Pathologe 2008 · [Suppl 2] 29:104-105

DOI 10.1007/s00292-008-1092-1

Online publiziert: 9. Oktober 2008

(c) Springer Medizin Verlag 2008

\author{
M. Dietel \\ Institut für Pathologie, Charité-Universitätsmedizin Berlin, Campus Mitte
}

\title{
Eröffnungsrede des Vorsitzenden der Deutschen Gesellschaft für Pathologie
}

Verehrte Mitglieder der Deutschen Gesellschaft für Pathologie,

sehr verehrte Gäste,

meine sehr verehrten Damen und Herren,

hiermit möchte ich Sie herzlich zur Eröffnungsveranstaltung der 92. Jahrestagung der Deutschen Gesellschaft für Pathologie und des 8. Bundeskongresses Pathologie Berlin des Berufsverbandes Deutscher Pathologen, d. h. also zur zweiten Woche der Pathologie, hier in der Ruine des historischen Rudolf-Virchow-Hörsaales begrüßen. Die Woche der Pathologie findet wieder in enger Zusammenarbeit mit der Deutschen Sektion der „International Academy of Pathology" statt, sodass die drei deutschen Pathologieorganisationen gemeinsam vertreten sind.

Ich freue mich, dass Sie so zahlreich gekommen sind und dass wir bei diesem herrlichen Wetter einige entspannte Stunden zusammen verbringen können.

Meine Ausführungen heute möchte ich ganz bewusst unter das Motto stellen:

Pathologie heute - es ist eine Freude dabei zu sein!

Bei dieser etwas euphorischen Aussage, die für einen Norddeutschen eigentlich untypisch ist, stellt sich naturgemäß sofort die Frage, warum?

Die Antwort ist leicht und lautet dahingehend: Die aktuellen wissenschaftlichen Entwicklungen in der molekularen Infektions- und Tumorpathologie sind so stürmisch, diagnostisch bedeutsam und patientenrelevant, dass wir Pathologen heute ein höheres diagnostisches Niveau erreichen und unser Fach wieder sehr viel mehr in das
Zentrum der klinischen Diagnostik und bemerkenswerterweise auch ins Zentrum der therapeutischen Onkologie und Infektiologie gerückt ist.

Die moderne Onkologie entwickelt sich in rasanten Schritten in Richtung einer zielgerichteten individualisierten Therapie. Dabei binden und hemmen hochspezifische Medikamenten zielgenau bestimmte molekulare Strukturen, so genannte „molecular targets", um durch Blockade der zugehörigen Signalwege z. B. das Tumorwachstum zumindest zu verlangsamen oder ganz zum Stillstand zu bringen. Voraussetzung für den Erfolg dieser so genannten ,targeted therapy“ ist der Nachweis der Zielstrukturen im individuellen Tumorgewebe. Dieser Nachweis geschieht in der aktuellen Medizin praktisch ausschließlich durch den Pathologen. Die gewebebasierten Untersuchungen, sei es durch Immunhistologie, In-situ-Hybridisierung oder molekulare Analysen, wie spezifische Sequenzierungen usw., sind Aufgaben der Pathologie. Es findet damit ein Paradigmenwechsel statt: Der Pathologe stellt nicht nur die Diagnose Krebs, sondern trägt darüber hinaus dazu bei, die individuelle Therapie, sprich die Gabe z. B. von Panitumumab oder Herceptin, festzulegen. Und dies ist erst der Anfang - man denke z.B. nur an Bronchial-, Plattenepithel- und Urothelkarzinome, für die in absehbarer Zeit auch zielgerichtete Medikamente vorliegen werden. Auch für diese müssen jeweils diagnostische Tests durch die Pathologie entwickelt werden. Der Pathologe hat also einen entscheidenden Einfluss auf die Therapie des einzelnen Menschen. Gleiches gilt auch für die Infektionspathologie, da in der einzel- nen Gewebeprobe die Erreger, z. B. Mykobakterien, verschiedene Viren usw., nachgewiesen werden können. Auf diesem Ergebnis baut dann die spezifische antiinfektiöse Therapie auf.

Diese aus unserer Sicht sehr erfreuliche und außerordentlich spannende Entwicklung ist - das möchte ich ausdrücklich betonen - nur deshalb möglich gewesen, weil sich die Pathologie in den letzten Jahren intensiv wissenschaftlich mit molekularen Gewebeanalysen und den damit verbundenen Techniken beschäftigt, diese weiterentwickelt und an die Bedingungen fixierter und paraffineingebetteter Proben adaptiert hat. Ganz wichtig dabei ist, dass die moderne molekulare Diagnostik, nach primärer Entwicklung an der Universität, in die Breite der diagnostischen Pathologie getragen wird und dass es in vernetzter Kooperation gelingt, eine flächendeckende Diagnostik sicherzustellen. Hierfür ist von entscheidender Bedeutung, dass die Deutsche Gesellschaft für Pathologie, der Berufsverband sowie die „International Academy of Pathology“ eng zusammenarbeiten, um auf allen drei Ebenen die entsprechende Expertise zu entwickeln und zu vermitteln.

Es sind seit vielen Jahren zahlreiche wissenschaftliche Aktivitäten unternommen worden, um dem fixierten menschlichen Gewebe möglichst viele Informationen zu entlocken und dabei gleichzeitig die Morphologie, d. h. die Zuordnung der einzelnen Analyseprodukte zu bestimmten Strukturen der Zelle, zu erhalten. Hätte die Pathologie nicht diese bemerkenswerte und von nicht Wenigen kritisch gesehene bis belächelte wissenschaftliche Arbeit ge- 
leistet, so wäre die zielgerichtete Therapie in den Kinderschuhen stecken geblieben. Es wäre schwierig bis unmöglich gewesen, die geeigneten Zielstrukturen der Medikamente in den Geweben überhaupt zu identifizieren.

Mit dieser Aufgabenerweiterung der $\mathrm{Pa}$ thologie, ist allerdings - und dies sei hier ebenfalls ausdrücklich betont - nicht nur eine große Chance, sondern auch eine große Herausforderung verbunden. Diese bezieht sich speziell auf reproduzierbare Qualitätsstandards und deren Kontrolle sowie die Konstanz und Transparenz der diagnostischen Prozesse in unseren Laboratorien. Dazu werden die Abläufe in den Laboratorien einem höheren Maß der Automatisierung zugeführt und die ärztliche Diagnosefindung vermehrt von Algorithmen geprägt werden müssen. Hieran gemeinsam mit der medizintechnischen Industrie zu arbeiten, halte ich für absolut essenziell, da ansonsten von benachbarten Diagnostikfächern leicht der Hinweis, oder nennen wir es durchaus Vorwurf, gemacht werden könnte, dass bei uns keine qualitätskontrollierten, standardisierten Prozesse ablaufen, sondern jeder letztlich so entscheidet, wie er will. Dieser Gefahr müssen wir begegnen, indem wir die oben genannten Maßnahmen proaktiv einleiten. Es muss jedem Pathologen klar sein, dass das Ergebnis der Gewebeuntersuchung einen direkten Einfluss darauf hat, ob ein Patient dieses oder jenes Medikament bekommt und damit länger oder kürzer lebt.

Das neue Aufgabenfeld der prädiktiven Pathologie wird vermutlich auch zu einer erhöhten öffentlichen Wahrnehmung führen - eine Herausforderung, der wir uns ebenso stellen müssen. Seit Jahren, wenn nicht seit Jahrzehnten, beklagen die Pathologen, dass die öffentliche Wahrnehmung mangelhaft ist, dass selbst Gesundheitspolitiker nicht wissen, was ein Pathologe macht, dass er z. B. die Diagnose Krebs stellt, und dass die Pathologie ganz wesentlich an zahlreichen wissenschaftlichen Entwicklungen teilgenommen hat, wie - ich komme damit auf den Ausgangspunkt zurück - die zielgerichtete Krebstherapie.

Um die öffentliche Wahrnehmung darüber hinaus zu stärken und für die Pathologie nutzbar zu machen, sind meines Ermessens verschiedene Maßnahmen notwendig:
1. Zunächst einmal muss die Pathologie als gemeinsame Institution auftreten. Es macht keinen Sinn, dass sich die Deutsche Gesellschaft, der Berufsverband und die Internationale Akademie für Pathologie getrennt an die Medien wenden, um eine neue Botschaft zu überbringen. Dies muss zukünftig gemeinsam geschehen. Als Nebeneffekt ist anzumerken, dass auch nur dann eine professionelle Medienarbeit möglich ist.

2. Aufgrund des neuen Aufgabenfeldes der Pathologie werden sich die Patienten zunehmend fragen: Wer ist eigentlich der Arzt, d. h. der Pathologe, der durch irgendwelche Untersuchungen entschieden hat, ich bekomme Herceptin, oder ich bekomme es nicht?

Wir werden zwangsläufig eine Öffnung in Richtung patientenorientierter Tätigkeit vornehmen müssen, oder - ich würde es lieber so formulieren - vornehmen dürfen. Ich glaube, es ist eine große Chance für uns, das Fach Pathologie in das Zentrum der medizinischen Diagnostik zurückzuführen, indem wir den Patienten gegenüber sehr viel offener auftreten. Erste Schritte in diese Richtung sind vor kurzem bei einem Treffen der amerikanischen und europäischen Pathologen getan worden, indem unter dem Motto „Pathologie für Patienten" ein webbasiertes Lexikon erstellt werden soll, das den Patienten ermöglicht, unsere Befunde zu verstehen. Diese Aktivität ist mittlerweile von der europäischen Gesellschaft für Pathologie und der „US-Canadian Association of Pathologists“ in den Grundzügen bestätigt worden und soll im nächsten Jahr umgesetzt werden. Aus meiner Sicht ist dieses eine sehr positive, aber auch sehr notwendige Aktivität, die den Patienten direkt oder indirekt die Bedeutung des Pathologen vermittelt. Eine ähnliche Aktivität führen wir zurzeit testweise hier an der Charité durch, indem wir eine Sprechstunde für Patienten mit Mammakarzinom eingerichtet haben, die, wenn sie im hiesigen Mammazentrum operiert worden sind, nach der Operation mit einem Pathologen über den Befund, mit Demonstration am Mikroskop, sprechen können. Diese Aktivität wird bisher allerdings nur zögerlich angenommen, aber viele der Patientinnen, die sie genutzt haben, sagen: „Jetzt habe ich es zum ersten Mal verstanden."

Der letzte Punkt, der kurz angesprochen werden soll und der eine gewisse Belastung für das Fach Pathologie darstellt, ist der nicht ausreichende Nachwuchs. Wir können feststellen, dass junge Kollegen an den Universitäten in großer Zahl das Fach Pathologie wählen, dass wir bei Anfängern an sich keine größeren Probleme haben, dass aber die so genannte „Drop-out-Rate“ relativ hoch ist, da Pathologen gut ausgebildete Mediziner mit interdisziplinärem AllroundWissen sind. Diese sind naturgemäß attraktiv für zahlreiche andere Aufgaben im Gesundheitssystem, und daher ist die Abwanderungsrate hoch. Es ist somit zwingend erforderlich, dass die Pathologie ihre Visibilität und ihre Attraktivität auch gegenüber den Medizinstudenten und jungen Medizinern wieder erhöht und aktiv betreibt, indem z. B. gezielte Förderung mit entsprechenden Karrierechancen und letztlich auch finanziellen Möglichkeiten angeboten wird. Die Deutsche Gesellschaft für Pathologie hat dafür eine spezielle Arbeitsgruppe gebildet, die von Herrn Prof. Schirmacher (Heidelberg) geleitet wird und sich mit der zukünftigen Entwicklung in diesem Bereich befassen wird.

Meine Damen und Herren, ich hoffe, dass ich Ihnen deutlich machen konnte, warum es eine Freude ist, zurzeit Pathologe zu sein, und dass diese Freude auf wissenschaftlichen Ergebnissen basiert, also aufgrund der Arbeit, die wir alle in vielen Stunden gemeinsam geleistet haben. Ich hoffe sehr, dass sich das positive Szenario, welches ich skizziert habe, in den nächsten Jahren in dieser Richtung weiter entwickelt, um insbesondere die Krebstherapie zusammen mit den klinischen Kollegen voranzubringen.

\section{Manfred Dietel}

\section{Korrespondenzadresse \\ Prof. Dr. Dr. h.c. M. Dietel}

Institut für Pathologie, Charité-Universitäts-

medizin Berlin, Campus Mitte

Charitéplatz 1, 10117 Berlin

manfred.dietel@charite.de 